\title{
Editorial
}

\section{Mathematical and Numerical Modeling of Flow and Transport}

\author{
Shuyu Sun, ${ }^{1}$ Eleanor W. Jenkins, ${ }^{2}$ \\ Zhangxing (John) Chen, ${ }^{3}$ and Juergen Geiser ${ }^{4}$ \\ ${ }^{1}$ Computational Transport Phenomena Laboratory (CTPL), Division of Physical Sciences and Engineering \\ (PSE), King Abdullah University of Science and Technology (KAUST), Thuwal 23955-6900, Saudi Arabia \\ ${ }^{2}$ Department of Mathematical Sciences, Clemson University, Clemson, SC 29634-0975, USA \\ ${ }^{3}$ Schulich School of Engineering, University of Calgary, 2500 University Drive NW, Calgary, \\ AB, Canada T2N $1 N 4$ \\ ${ }^{4}$ Department of Mathematics, Humboldt-University of Berlin, Unter den Linden 6, 10099 Berlin, Germany
}

Correspondence should be addressed to Shuyu Sun, shuyu.sun@kaust.edu.sa

Received 19 August 2011; Accepted 19 August 2011

Copyright (c) 2011 Shuyu Sun et al. This is an open access article distributed under the Creative Commons Attribution License, which permits unrestricted use, distribution, and reproduction in any medium, provided the original work is properly cited.

\section{Introduction}

Most processes in natural and engineered systems inherently involve flow and transport. From atoms to galaxies, from inorganic reactions to living organisms, from oceans to streambeds, flow and transport processes occur across a wide range of physical applications. Application areas include groundwater contamination, carbon sequestration, air pollution, petroleum exploration and recovery, weather prediction, drug delivery, material design, chemical separation processes, natural disaster assessments, and others. As a result of its importance in a variety of problems of interest, accurate and efficient numerical modeling of flow and transport is essential.

Interest in the numerical simulation of flow and transport mechanisms has increased in recent years along with the rapid increase in computational power. Spectacular advances in computer performance and emerging technologies of parallel distributed grids and GPU computing have provided flow and transport modelers with tools to simulate and study complex phenomena. However, efficient numerical algorithms, new mathematical formulations, and advanced computational techniques are required if researchers are to take full advantage of this progress and address new and existing challenges in flow and transport modeling and simulation. One particular challenge requires modelers to synthesize varied temporal and spatial scales at which flow and transport mechanisms operate. Nanoscale 
changes tend to be fast, while geological changes occur over much larger time scales. Thus, algorithms that are optimal for one problem set need not be so for another. The physics associated with each process and often with each of the spatial and temporal scales are coupled, requiring that one take a global approach to developing appropriate numerical algorithms. For example, a numerical algorithm for a flow process should conserve mass locally, but it is imperative that this happen if the flow model is to be coupled to a transport model. Temporal integration methods for nanoscale simulations must be incorporated into the integration method used for larger scales. The list goes on.

\section{Overview of Work Presented in this Special Issue}

The papers presented in this special issue cover state-of-the-art simulations of flow and transport problems; they represent ongoing research projects on various important topics relevant to the modeling and computation of flow and transport. In particular, this special issue focuses on four important areas of complex flow and transport: (1) porous media flow and transport, (2) coupling of flow and transport to heat transfer, (3) flow and transport in chemical, biological, and sociological systems, and (4) new numerical algorithms to tackle challenges in flow and transport simulation. We outline each of the four areas below.

Porous media flow and transport is of great community interest because of its importance in oil production and environmental protection. A group of papers addresses various issues in this area. The paper entitled "Numerical simulation of pollutant transport in fractured vuggy porous karstic aquifers" contains an analysis of a mathematical model for contaminant transport in fractured vuggy porous media. The results show how different distributions of vugs and fractures impact the contaminant transport and the effluent outlet concentration. In the paper entitled "A parallel stochastic framework for reservoir characterization and history matching," the authors consider the spatial distribution of parameters that characterize subsurface porous media, and they present an efficient, accurate, and parallel framework for estimating reservoir parameters. The methodology uses measurement data such as inferred phase pressures, phase concentrations, fluxes, and seismic and well-log data. The paper entitled "An analytical solution of the advection dispersion equation in a bounded domain and its application to laboratory experiments" is a study of the modeling of contaminant transport through a saturated porous medium under a uniform flow in a parallel plate geometry. A general solution of the advection dispersion equation in a porous medium is provided and used to examine experimental data acquired via a noninvasive technique. In "Effects of gravity and inlet/outlet location on a two-phase cocurrent imbibition in porous media," the authors use a two-dimensional simulation tool to numerically investigate gravity and inlet/outlet location effects on two-phase cocurrent imbibition in a porous medium. Three different cases of side-, top-, and bottom-inlet location are considered.

Another group of papers contains analyses of flow and transport coupled with heat transfer. Incorporating heat transfer into a flow and transport system usually requires the addition of an energy balance equation. Models of fully coupled flow, transport, and heat transfer are useful in heat transfer enhancement technology, where, for example, transversal turbulators are used to enhance the heat transfer rate by reducing the thermal resistance near surfaces. The paper entitled "Numerical analysis of water forced convection in channels with differently shaped transverse ribs" addresses this particular application with a numerical investigation of turbulent water forced convection in a ribbed channel. A very realistic case study is included in the paper entitled "Numerical study of the effect of a power plant cooling water 
discharge in the Montevideo Bay," where the authors present a finite-element based numerical simulation of the effects of power plant cooling water on the overall water temperature in the Río de la Plata River and Montevideo Bay. Simulations of thermomagnetic convection in a differentially heated square cavity using the single relaxation time lattice Boltzmann method are the focus of the paper entitled "Simulation of thermomagnetic convection in a cavity using the Lattice Boltzmann model." These models are important in understanding the cooling processes associated with microelectronic devices, which are becoming more ubiquitous each day. The paper entitled "Radiative mixed convection over an isothermal cone embedded in a porous medium with variable permeability" contains an analysis of the interaction of mixed convection with thermal radiation in an optical dense viscous fluid adjacent to an isothermal cone imbedded in a porous medium.

There is also a group of papers that addresses flow and transport mechanisms in application areas associated with chemical, biological, and sociological systems. In these complex systems, flow and transport often interact with other phenomena. In "Modeling and simulation of a chemical vapor deposition," the authors consider a chemical system and propose a model on PE-CVD (plasma-enhanced chemical vapor deposition) processes for metallic bipolar plates. They also discuss the optimization of the processes for deposition of a heterogeneous layer of material on a metallic plate. A biological system is investigated in "Numerical simulation of solid tumor blood perfusion and drug delivery during the "vascular normalization window" with antiangiogenic therapy," where the authors report on the influence of vascular normalization on solid tumor blood perfusion and drug delivery using a generated blood vessel network for simulations. A stochastic approach for estimating freeway capacity is the focus of "Implementing estimation of capacity for freeway sections." New approaches for predicting freeway capacities are necessary as demands on transportation systems increase in proportion to population densities. The subject of the paper entitled "Modeling and analysis of material flows in re-entrant supply chain networks using modified partial differential equations" is material flow in a supply chain. Supply chain networks are of great interest to industry, but their study is complicated by human interaction with the network. The authors begin the paper by reviewing the basic partial differential equation models for supply chain networks and follow by proposing a modified continuum model for reentrant supply chains. They demonstrate the usefulness of their model using numerical examples.

There is a group of papers that addresses advanced numerical methods, which are vital to resolving the many challenges associated with flow and transport simulation. In " $A$ stabilized mixed finite element method for single-phase compressible flow," the authors present and study a stabilized mixed finite element method for single-phase compressible flow through porous media. A moving particle solution for the Navier-Stokes equations is considered in "Development of a particle interaction kernel function in MPS method for simulating incompressible free surface flow." The authors study a kernel function that accounts for the interaction among moving particles within the particle method framework. In "Multiscale time-splitting strategy for multiscale multiphysics processes of two-phase flow in fractured media," a multiscale time splitting strategy to simulate multiscale multiphysics processes of two-phase flow in fractured porous media is proposed and analyzed. This is especially useful when handling rapid changes of the pressure and saturation normally associated with fractured systems with capillarity. In "Adaptive optimal m-stage Runge-Kutta methods for solving reaction-diffusionchemotaxis systems," the author proposes an adaptive optimal time stepping strategy for the explicit m-stage Runge-Kutta method to solve reaction-diffusion-chemotaxis systems. "Computing exponential for iterative splitting methods: algorithms and applications" contains 
a study of more general iterative splitting methods, and the author proposes efficient algorithms to solve the splitting schemes.

\section{Conclusions}

The progress in understanding flow and transport and their interaction with other physical, chemical, biological, and sociological processes is strongly dependent on adequacy, accuracy, and efficiency of numerical modeling and simulation. This special issue focuses on four important areas of flow and transport and it highlights new numerical algorithms to address conventional challenges of flow and transport simulation. The issue is not intended to be an exhaustive collection nor a survey of all of the current trends in flow and transport research; many additional significant research areas of flow and transport exist, and include, for example, coupling of different flows (e.g., coupling Stokes and Darcy flows), coupling of flow and transport in different scales (e.g., transport in molecular scale, pore scale, lab scale, and field scale), information flow (as opposed to material flow), and nonlinearity in flow and transport (non-Newtonian fluid, non-Darcy flow, etc.). Space limitations have prevented these important topics from being included here, but our hope is that they will be addressed in future special issues.

\section{Acknowledgments}

The authors would like to thank the participants of the special issue for their inspiring contributions, and the anonymous reviewers for their diligent work, which led to the high quality of the special issue. The Lead Guest Editor (S. Sun) would like to acknowledge KAUST faculty Baseline Research Fund (BRF) for supporting his research in flow and transport.

Shuyu Sun

Eleanor W. Jenkins

Zhangxing (John) Chen

Juergen Geiser 


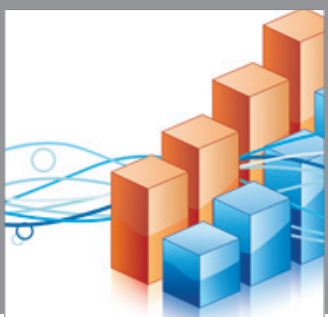

Advances in

Operations Research

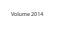

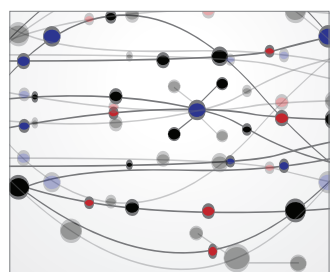

\section{The Scientific} World Journal
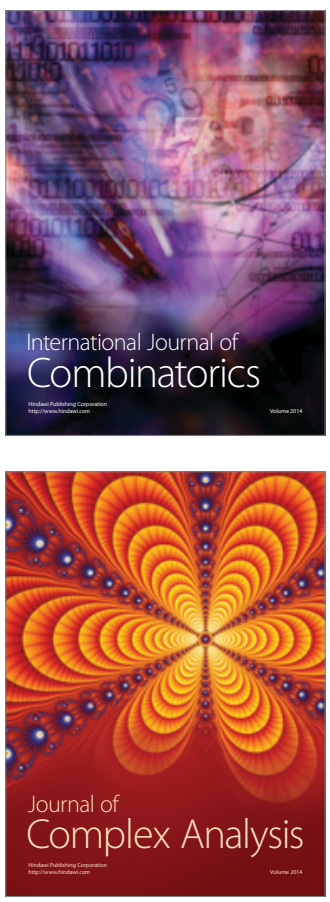

International Journal of

Mathematics and

Mathematical

Sciences
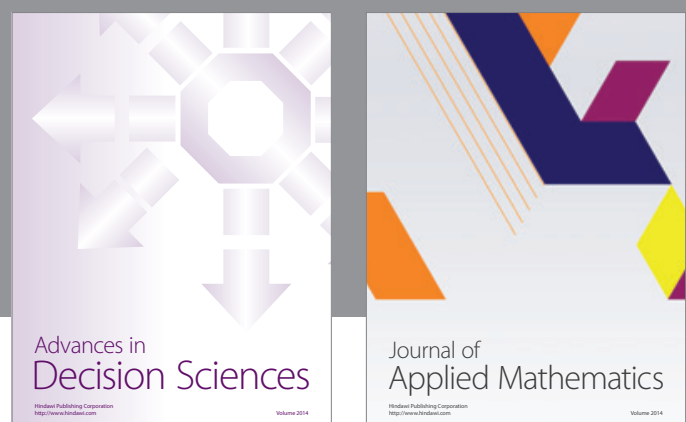

Journal of

Applied Mathematics
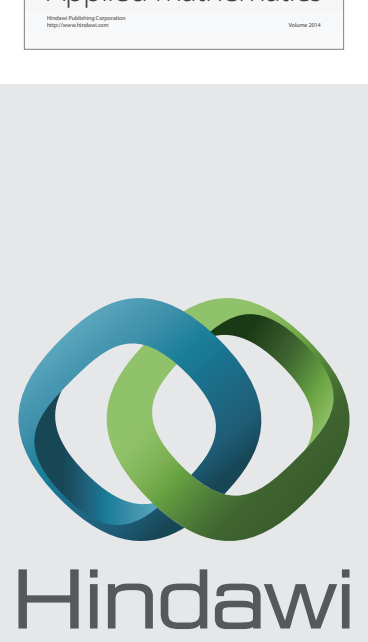

Submit your manuscripts at http://www.hindawi.com
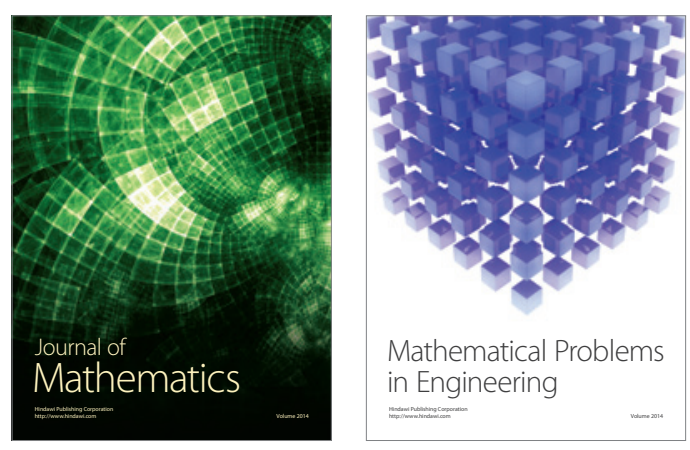

Mathematical Problems in Engineering
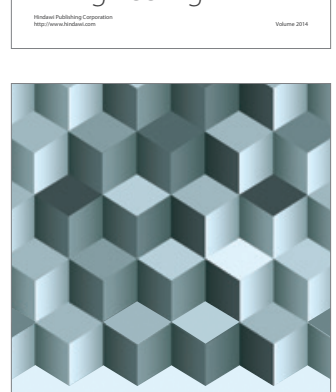

Journal of

Function Spaces
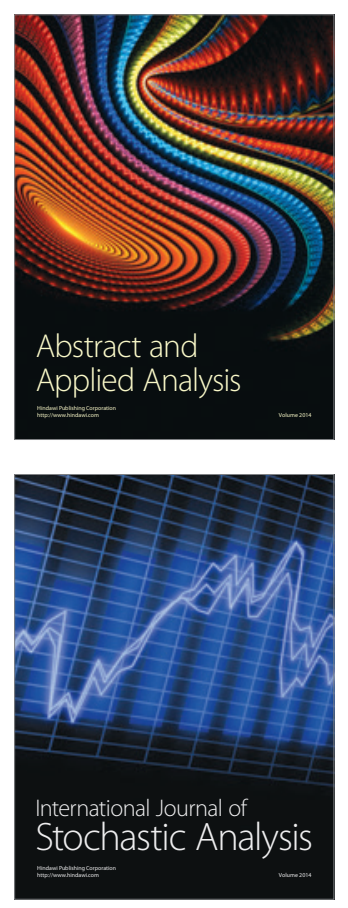

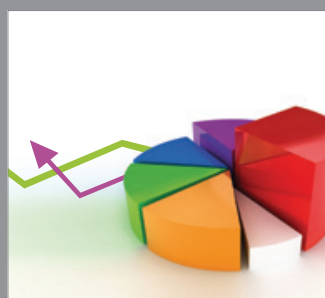

ournal of

Probability and Statistics

Promensencen
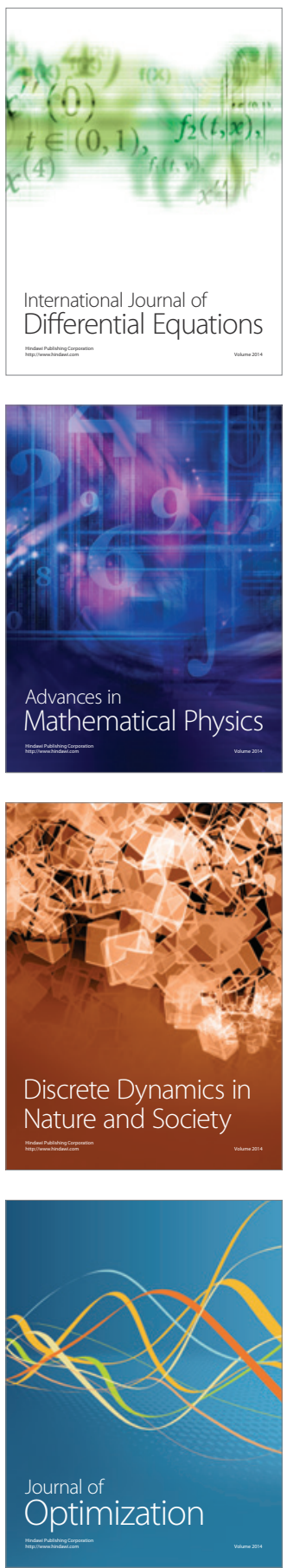